\title{
AN Alternative APPROACH FOR SELECTION OF PSEUdo RANDOM Numbers FOR ONLINE EXAMINATION SYSTEM
}

\author{
Shilpi Kumari Shaw ${ }^{1}$, Aakash Sharma ${ }^{2}$, Shoubhik Chowdhury ${ }^{3}$, \\ Aritra Biswas ${ }^{4}$, Arnab Mitra ${ }^{5}$ \\ 1,2,3,4,5 Department of Computer Science \& Engineering \\ Adamas Institute of Technology, Barasat-700126, India \\ \{shilpishaw.ait, aakashcal91, shoubhikchowdhury91, \\ biswas.aritra8, mitra.arnab\} @gmail.com
}

\begin{abstract}
Fast and accurate selection of random pattern is needed for many scientific and commercial applications. One of the major applications is Online Examination system. In this paper, a sophisticated approach has been developed for the selection of uniform pseudo random pattern for Online Examination System. Three random integer generators have been compared for this purpose. Most commonly used procedural language based pseudo random number; PHP random generator and atmospheric noise based true random number generator have been considered for easy generation of random patterns. The test result shows a varying degree of improvement in the quality of randomness of the generated patterns. The randomness quality of the generated pseudo random pattern has been assured by diehard test suite. An experimental outcome for our recommended approach signifies that our approach selects a quality set of random pattern for Online Examination System.
\end{abstract}

\section{KEYWORDS}

Pseudo random number generator (PRNG), Pseudo random Pattern Generator (PRPG), Procedural Language Random Number Generator (PrRNG), PHP random Number Generator (PHPRNG), True Random Number Generator (TrRNG), Online Examination System (OES)

\section{INTRODUCTION}

Random number [1], [2] is a number engendered by a method, whose consequence is volatile, and which cannot be sub sequentially dependably replicated. It is purely impossible to authenticate whether the certain number was formed by a random number generator or not. In order to study the predictability of the output of such a producer, it is therefore unconditionally essential to reflect order of number. It is pretty direct to express whether an order of infinite extent is random or not. This arrangement is random if the amount of info it covers - in the logic of Shannon's information concept - is also infinite. In additional falling out, it must not be probable for a processer, whose extent is finite, to produces this arrangement. Excitingly, an infinite random arrangement comprises all probable predetermined sequences. Such an unbounded arrangement does for instance hold the Microsoft Windows source code or the copy of the Geneva 
conventions. Unfortunately, this description is not very worthwhile, as it is not promising in exercise to create and practice infinite orders.

In the case of a finite arrangement [3] of figures, it is validly difficult to confirm whether it is random or not. It is only conceivable to check that it shares the statistical stuffs of a random arrangement- similar to equi-probability of the whole numbers - but this is a challenging and complex task. To demonstrate this, let us for specimen consider a binary random number generator constructing sequences of ten bits. Although it is precisely as probable as any other ten bits sequences, 0000000000 does look a lesser random than 1001010111 .

Pseudo-random number generators (PRNGs) [4], [5] are algorithms to instinctively produce elongated runs of numbers with superior random properties however eventually the series repeats. The sequence of values evaluated by such algorithms is frequently resolute by a rigid number known as a seed [4]. The most frequent PRNG is the linear congruential generator, which utilizes the recurrence in Equation 1.

$$
A_{n+1}=\left(c^{*} A_{n}+d\right) \bmod m \ldots . .(1)
$$

where $A$ is the sequence of pseudorandom values, and

$m, 0<m-$ the "modulus";

$c, 0<c<m-$ the "multiplier";

$d, 0 \leq d<m$ - the "increment";

$A_{0}, 0 \leq A_{0}<m-$ the "seed" or "start value".

There exist two major ways used to produce [6] random numbers. One processes certain physical phenomenon that is probable to be random and then recompenses for probable biases in the measurement procedure. The further practices computational algorithms that can yield long arrangements of apparently random outcomes, which are in fact absolutely determined by a smaller primary value, well-known as a seed. The latter forms are frequently known as pseudorandom number generators.

Many extents of statistical investigation, research, and simulation depend on the superiority of random number generators. Maximum programs for statistical data analysis comprise a function for producing uniform random numbers. The Diehard suite [5] of tests has grown into a standard technique of evaluating the superiority of uniform random number generator procedures

Diehard tests [5], [7-8] are a battery of statistical test to measure the standard of random number generated. At first it was developed by George Marsaglia and first issued in 1995 on a CD-ROM of random numbers. Soon after advanced version of this statistical test been reformed and circulated thru University of Hong Kong.

The below mentioned tests are performed to extent the feature of the randomness of an individual random pattern creator:

i. Birthday spacings: The name birthday spacing is centred on birthday paradox. Here random points are selected on a large interval with the asymptotically exponentially distributed spacing among the points.

ii. Overlapping permutation: This test always analyse five successive random number arrangements, with statically identical probability, a total 120 possible ordering should occur.

iii. Ranks of matrices: choose few number of bits from certain amount of random number to formulate a matrix over $[0,1]$, then govern the rank of the matrix on the basis of determinant value of matrix.

iv. Monkey tests: The name monkey test is based on infinite monkey theorem. In this test sequence of few numbers of bits is considered as "words". Therefore overlapping word 
present in a stream be counted. Any known distribution be followed by the number of "words" that don't appear.

v. Count the 1's: In this test, count the 1bit in every single of either successive or chosen bytes. Then convert the counted values into "letters" and lastly following the counting of the existences of five-letter 'words".

vi. Parking lot test: Unit circle in a $100 \times 100$ square placed randomly is tested to search whether any of the circles, overlaps a present one. After a repetition of 12000 tries, the number of effectively "parked" circles would follow a certain normal distribution.

vii. Minimum distance test: In this test, place randomly 8000 points in a $10.000 \times 10.000$ squares, after that minimum distance between the pair is to be found. The square of the distance with a certain mean is exponentially distributed.

viii. Random spheres test: In this test, in a cube of edge 1,000, choose randomly 4,000 points. On each point a sphere is to be centred, whose radius is the least distance to another point.

ix. The smallest sphere's volume ought to be exponentially distributed using a certain mean.

x. The squeeze test: Until one reach 1 , multiply $2^{31}$ by random floats on $[0,1)$. Repeat this thing 100,000 times. The number of floats required to arrive at 1 should pursue a certain distribution.

xi. Overlapping sums test: Create a extended series of random floats on [0,1]. Add series of 100 successive floats. The sums have to be normally distributed with characteristic mean with sigma.

xii. Runs test: create a extended sequence of random floats on $[0,1)$. Count up ascending and descending runs. The counts have to pursue a certain distribution.

xiii. The craps test: Count the success and the number of toss per game, play 200,000 games of craps. Each count ought to pursue a certain distribution.

The organization of this paper is as follows: Section 2 briefly discusses the related work. Section 3 expounds the proposed work. Section 4 records the experimental results. Section 5 draws the conclusion. Section 6 is devoted to acknowledgements, and Section 7 includes references.

\section{RELATED WORK}

A number of methods have been applied [9-13], [16] to generate better quality random numbers. Some novel labours have been established to produce better quality random numbers. Some significant efforts have also been made to produce pseudo-random numbers. Among all Intel true random number and true random number generation by Random.org is pretty remarkable where atmospheric noise is applied as the seed.

A true random number[14], [15] producer is a section of electronics that plugs inside a computer and yields genuine random numbers as disparate to the pseudo-random numbers that are created by a computer program. The standard technique is to intensify noise produced thru a resistor (Johnson noise) or a semi-conductor diode and feed this to a Schmitt trigger or comparator. If one samples the out-come (not too rapidly) get a sequence of bits which are statistically autonomous.

Utmost random numbers [4] applied in computer programs are pseudo-random, which means they are created in a expectable fashion via a mathematical formulation. This is adequate for several purposes; however it might not stay random in the way one imagines if it used to gamble like dice rolls and lottery drawings.

RANDOM.ORG [16], [17] proposed true random numbers to any person on the Internet. The randomness originates from atmospheric noise, which for numerous drives is superior to the pseudo-random number algorithms usually applied in computer programs. For gambling sites, for scientific applications and for art and music people use RANDOM.ORG. 


\section{Proposed Work}

Generation of our algorithm is to obtain random question number in on-line examination system using PHP. Compare to other similar on-line examination system this system is more innovative because of the generation of pseudo random pattern, as a result none of the question get repeated at boundless instant of time.

Procedural language provides random number generation function rand(), which is found in $<$ stdlib.h> header. Function rand actually produces pseudorandom numbers. Calling rand recurrently produces a arrangement of numbers that seems to be random.

Based on the above mentioned, the following flowchart 1 and Algorithm 1, should generate the random integer set in procedural language.

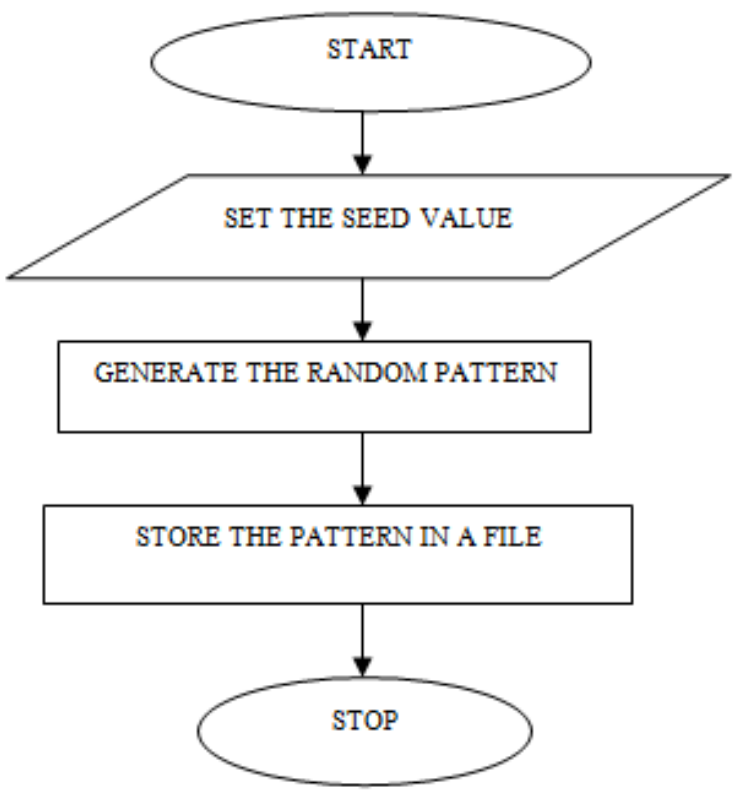

Figure 1: Flow chart for generating a random pattern in procedural language

Similarly just like Procedural Language random function, PHP also have its own random function mt_rand().The number generated in PHP is uniformly distributed over the specified range. PHP integers are 32 or 64 bits wide, and are represented by means of two's complement arithmetic.

Based on the above mentioned, the following flowchart 2 and Algorithm 2, should generate the random integer set in PHP. 


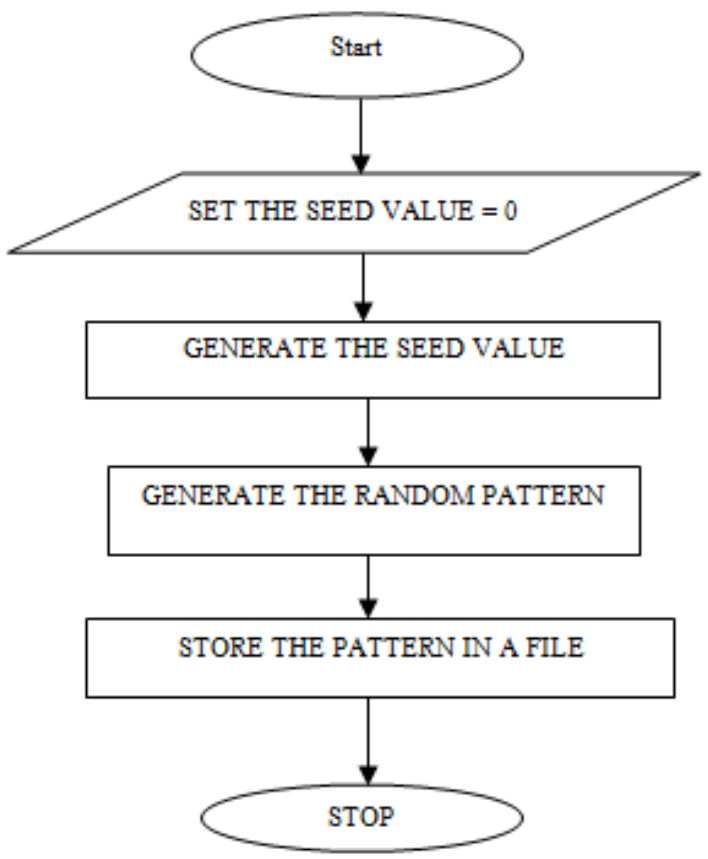

Figure 2: Flow chart for generating a random pattern in Scripting Language

Finally the proposed algorithm which has been proved to be better compare to previous algorithms, and gave better randomness quality. The following flowchart 3 and Algorithm 3 should generate the random integer set.



Figure 3: Flow chart for selecting a true random sequence for online examination 
Algorithm 1: Selection of Pseudo Random Pattern Sequence

Input: Set of true random sequence

Output: Random pattern for randomly generated question set

(Write this following algorithm in step wise only. no pseudocode)

STEP 1: Start

STEP 2: Generating a random number using the seed value.

STEP 3: According to the random number, the position has been found from the file in which the pseudo random patterns are stored.

STEP 3.1: Searching the random number from the file.

STEP 3.2: In the case of more than one digit number, the digits are concatenated with each other.

STEP 3.3: The total string has been converted into integer.

STEP 4: After matching the first element, insert the corresponding row elements into the array.

STEP 4.1: Insert the element into the first position of the array until a space is occurred.

Checks the next field is blank or a new line

If matched

The previous value has put into the array

STEP 4.2: Repeat the above step (STEP 4.2) until a new line or the end of the file

$(\mathrm{EOF})$ is reached.

STEP 4.3: The elements that are found in the row are converted to integer value.

STEP 5: Print the array elements of the selected row.

STEP 6: Get the selected random pattern sequence for randomly selected question set.

\section{STEP 7: End}

Our proposed above algorithm have generated random number up to 3 digits. That means it can generate up to 1000 random sequences which is much more than our requirement for online examination purpose, as we know in an exam session we hardly require 40/50 question. Further this algorithm is also efficient to produce random numbers larger than 3 digits on requirement, which has been shown in Figure 4(b).

\section{EXPERIMENTAL OBSERVATION AND RESUlt ANALYSIS}

The given file contains the random patters stored in text format.

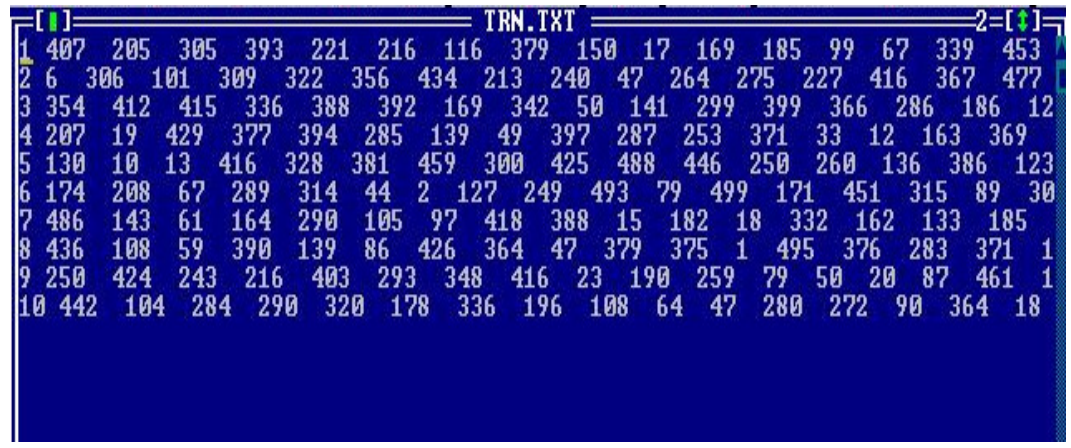

Figure 4(a): The text file in which the true random sequences are stored 


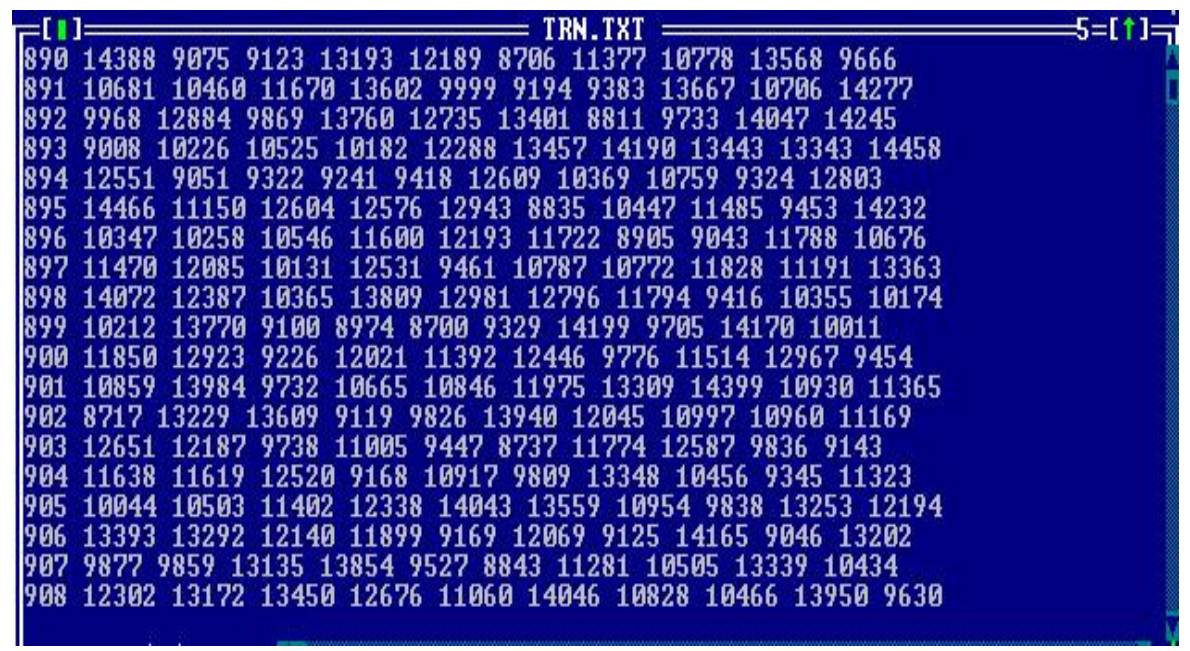

Figure 4(b): The text file in which the true random sequences are stored

The algorithm generates a random number between 0 to (seed value - 1), i.e. 0 - 9 in this example. It then starts searching from the beginning of the text file containing the random patterns. If the first number matches the random number, the entire row containing the random pattern is taken into an integer array else the row is scanned simply as text. The algorithm then scans the first element of the next row. If the number is found, it takes the random pattern into the array else continues searching. The algorithm continues until the random number is matched with the first element and the corresponding random pattern is selected.

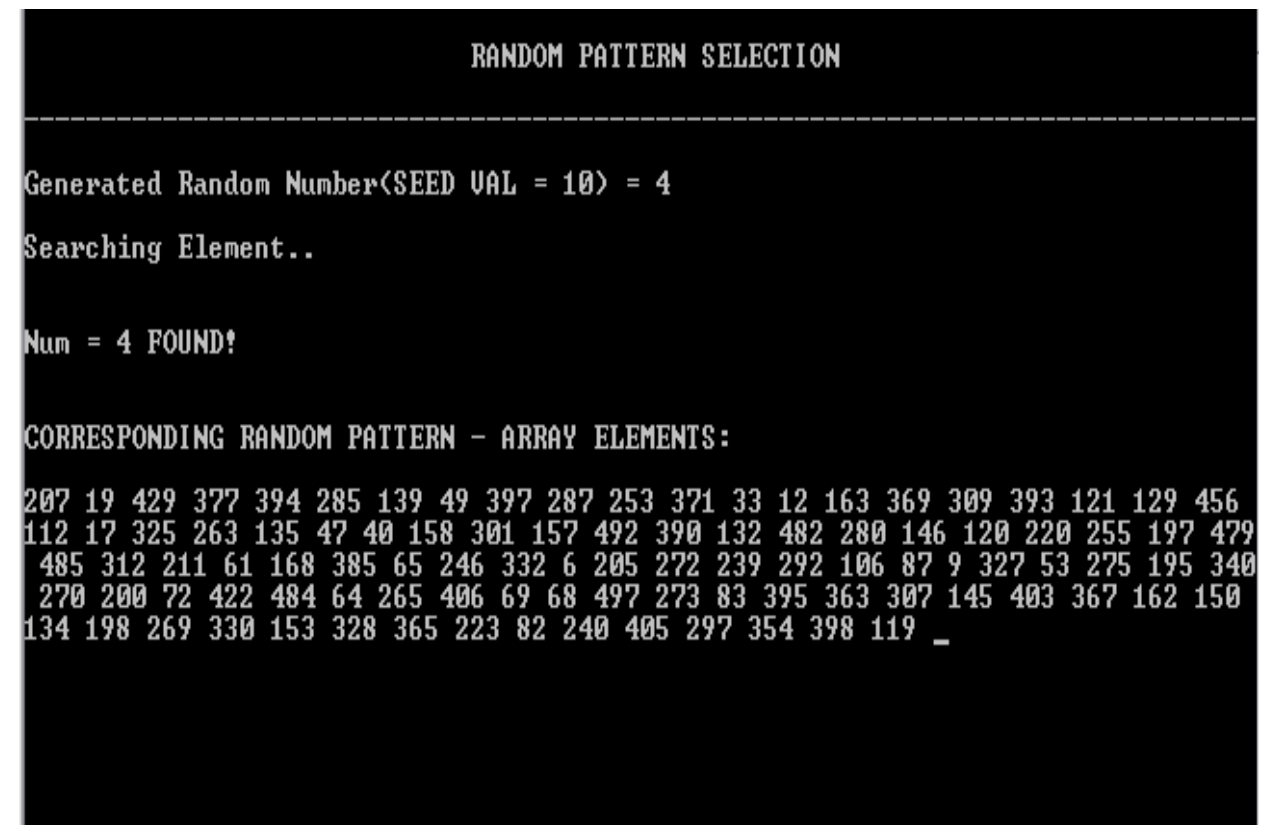

Figure 5(a): Outcomes of the Proposed Algorithm 


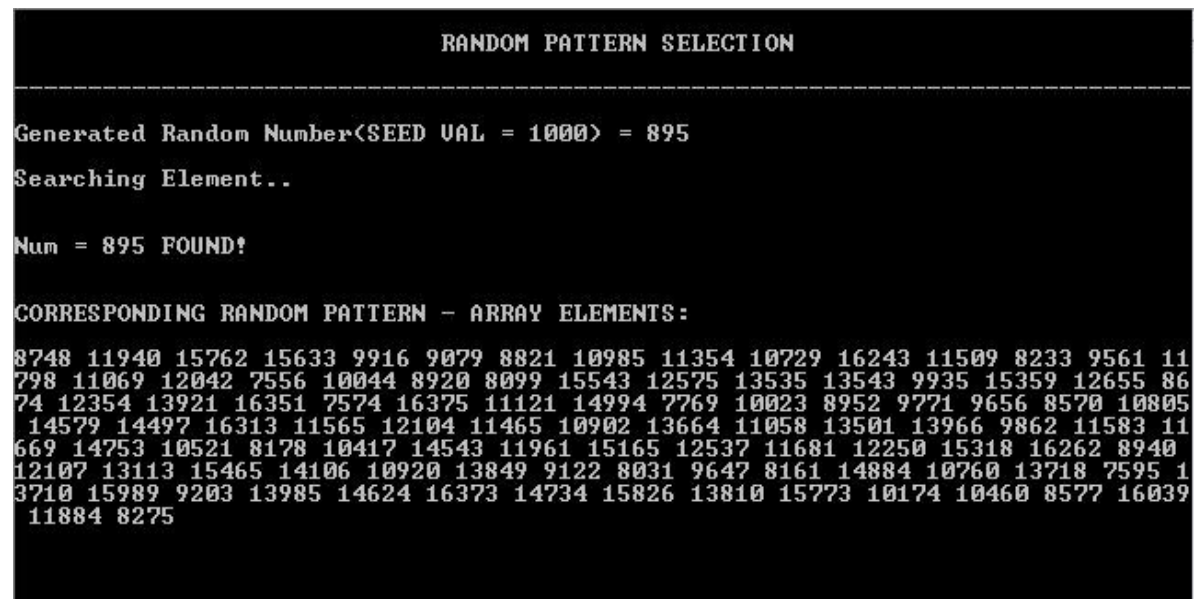

Figure 5(b): Outcomes of the Proposed Algorithm

Centred on this factor Table 2 replicates the end result of Diehard tests. The examined outcomes can be observed in Figure 4 with graph, which demonstrate the improved feature of randomness for resultant random integer originator

.Most of the tests in DIEHARD returns a p-value, which should be uniform on $[0,1)$ if the input file contains truly independent random bits. Those $p$-values are chosen up by $p=F(Y)$, where $F$ is the presumed distribution of the sample random variable $\mathrm{Y}$---often normal. But that presumed $\mathrm{F}$ is just an asymptotic approximation, for which the fit will be worst in the tails. Thus outcome should not be surprised with occasional p-values near 0 or 1 , such as .0012 or .9983 . When a bit stream really FAILS BIG, it will get p's of 0 or 1 to six or more places. By every means, the value of $\mathrm{p}<.025$ or $\mathrm{p}>.975$ means that the RNG has "failed the test at the .05 level".

Table 1: Diehard Test

\begin{tabular}{|c|l|}
\hline Test Number & \multicolumn{1}{|c|}{ Diehard Test Name } \\
\hline 1. & Birthday Spacings \\
\hline 2. & GCD \\
\hline 3 & Gorilla \\
\hline 4. & Overlapping Permutations \\
\hline 5. & Ranks of 31x31 and 32x32 matrices \\
\hline 6. & Ranks of 6x8 Matrices \\
\hline 7. & The Bitstream Test \\
\hline 8. & Monkey Tests OPSO,OQSO,DNA \\
\hline 9. & Count the 1`s in a Stream of Bytes \\
\hline 10. & Count the 1`s in Specific Bytes \\
\hline 11. & Parking Lot Test \\
\hline 12. & Minimum Distance Test \\
\hline 13. & Random Spheres Test \\
\hline 14. & The Sqeeze Test \\
\hline 15. & Overlapping sums Tests \\
\hline 16. & Runs Up and Down Test \\
\hline 17. & The Craps Test \\
\hline
\end{tabular}


Table 2: Test Result

\begin{tabular}{|c|c|c|c|}
\hline $\begin{array}{c}\text { Diehard Test } \\
\text { Number }\end{array}$ & $\begin{array}{c}\text { Procedural } \\
\text { Language }\end{array}$ & PHP & TRNG \\
\hline 1. & Fail & Fail & Pass \\
\hline 2. & Fail & Fail & Fail \\
\hline 3. & Fail & Fail & Fail \\
\hline 4. & Fail & Fail & Pass \\
\hline 5. & Fail & Fail & Fail \\
\hline 6. & Fail & Fail & Pass \\
\hline 7. & Fail & Fail & Fail \\
\hline 8. & Fail & Fail & Pass \\
\hline 9. & Fail & Fail & Pass \\
\hline 10. & Fail & Fail & Pass \\
\hline 11. & Fail & Fail & Pass \\
\hline 12. & Fail & Fail & Pass \\
\hline 13. & Fail & Fail & Pass \\
\hline 14. & Fail & Fail & Pass \\
\hline 15. & Fail & Fail & Pass \\
\hline 16. & Fail & Fail & Pass \\
\hline 17. & Fail & Fail & Pass \\
\hline $\begin{array}{c}\text { Total Number } \\
\text { of Diehard Test } \\
\text { Passes }\end{array}$ & 0 & 0 & 13 \\
\hline
\end{tabular}

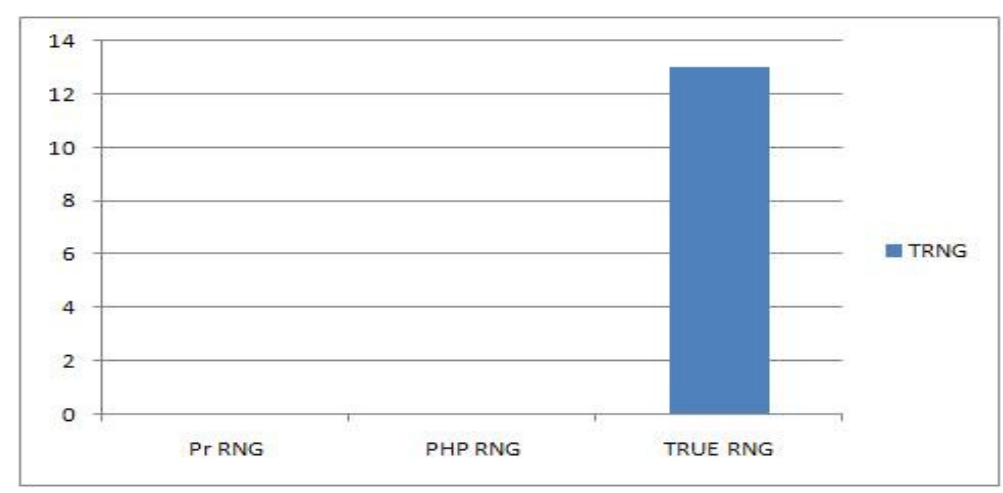

Figure: 6 Result of the above mentioned table is shown in the following graph.

The graph below show the randomness quality of a random pattern developed in $\mathrm{C}$ language, PHP, and proposed algorithm.

Procedural language has its own random function for generating random pattern, this function give a random pattern between 0 to 99, which is plotted in the given graph in yellow colour.

Similarly PHP has also its own random function for generating random pattern, its randomness is also plated in the same graph with pink colour.

But after comparing the proposed algorithm for random pattern generation with C's and PHP's random pattern generation function by plotting the randomness in the same graph with blue colour by taking different values, the outcomes shows that this proposed algorithm gives improved random quality. 
Outcomes based on figure 8 , it can be concluded that our proposed algorithm can be appliance as an enhanced source of random patterns as it have maximum amount of randomness with allusion to all further random function.



Figure 8: Randomness testing with higher number of data set

\section{CONCLuSion}

The algorithm selects a better random pattern and shows that the number selected from the pattern has no repetition. The graph represents as figure 2 express the superiority of randomness archived by this algorithm.

So as per our requirement, through this algorithm we can achieve random questions for our online examination system, and thus meets our requirements.

\section{REFERENCES}

[1] http://en.wikipedia.org/wiki/Random_Number

[2] Arnab Mitra, Anirban Kundu; (2012) Cost optimized Approach to Random Numbers in Cellular Automata; The Second International Conference on Computer Science, Engineering \& Applications (ICCSEA); India

[3] Wolfram; Wolfram Mathematica Tutorial Collection: Random Number .http://www.wolfram.com/learningcenter/tutorialcollection/RandomNumberGeneration/.pdf

[4] http://en.wikipedia.org/wiki/Pseudorandom_number_generator

[5] Arnab Mitra, Anirban Kundu; (2012) Cost Optimized Design Technique for Pseudo-Random Numbers in Cellular Automata; International Journal of Advanced Information Technology (IJAIT) Vol. 2, No.3, June 2012

[6] http://en.wikipedia.org/wiki/Random_Number_Generationen.wikipedia.org/wiki/Diehard_tests

[7] Robert G. Brown. dieharder: A Random Number Test Suite, 2006a.

[8] http://www.phy.duke.edu/ rgb/General/dieharder.php. C program archive dieharder, version 1.4.24.

[9] Colin Plumb; (1994) "Truly Random Numbers"; Dr.Dobbs Journal, November 1994, p.113.

[10] Tim Matthews; (1995) "Suggestions for random number generation in software"; RSA Data Security Engineering Report, 15 December 1995.

[11] Boaz Barak and Shai Halevi; (2005) An architecture for robust pseudo-random generation and applications to /dev/random; In Proc. ACM Conf. on Computing and Communication Security (ACM CCS); 2005.

[12] B. Jun and P. Kocher; (1999) The Intel Random Number Generator.; Cryptography Research Inc. white paper, Apr. 1999.

[13] Matsumoto, M. and Nishimura, T. (1998), "Mersenne-Twister: A 623-Dimensionally Equidistributed Uniform Pseudo-Random Number Generator," ACM Transactions on Modeling and Computer Simulation, 8:1, pp. 3- 30..

[14] http://en.wikipedia.org/wiki/True_Random_Number_Generator

[15] Dirk Eddelbuettel; Random: An $\mathrm{R}$ package for true random numbers; http://dirk.eddelbuettel.com/bio/papers.html

[16] www.random.org

[17] http:// www.random.org/randomness/ 